\title{
Inhaltsverzeichnis
}

\section{Holger Altmeppen}

Insichgeschäft und Stimmverbot am Beispiel der GmbH $\ldots \ldots \ldots \quad 1$

\section{Gregor Bachmann}

Der Bergmann-Senat Revisionsrechtsprechung im Gesellschaftsrecht am Anfang des 21. Jahrhunderts ........................

WALter BAyer

Einziehung von GmbH-Geschäftsanteilen:

Streifzug durch die Rechtsprechung des II. Zivilsenates des BGH

Falk Bernau

Unter welchen Voraussetzungen ist der an der betroffenen

Drittgesellschaft beteiligte GmbH-Gesellschafter vom Stimmrecht gemäß 47 Abs. 4 Satz 2 GmbHG ausgeschlossen? ............

Manfred Born

Erleichterung der Darlegungs- und Beweislast für ausgeschiedene Organmitglieder im Innenhaftungsprozess de lege lata? ...... 79

\section{Moritz Brinkmann}

Drahtseilakt ohne Sicherung?

Die Haftung aus den Zahlungsverboten nach $\$ \$ 64$ S. 1 GmbHG, 92 Abs. 2 AktG und die Deckung durch die D\&O Versicherung

Andreas Cahn

Kapitalmarktrechtliche Aspekte von Zusammenschluss-

und Akquisitionsvorhaben

Matthias Casper

Die Beseitigung geheilter Beschlüsse

nach $\$ 242$ Abs. 2 S. 3 AktG -

Weckruf für eine Norm im Dornröschenschlaf $\ldots . \ldots \ldots \ldots$.

Meinrad Dreher

Die D\&O-Individualversicherung 
INGo Drescher

Die schwebende Nichtigkeit ........................ 169

Holger Fleischer

Der Gesellschaftsvertrag der Fugger:

Frühform des OHG-Rechts

BARBARA GrüNEBERG

$\$ 28$ Abs. 1 HGB bei der vermögensverwaltenden

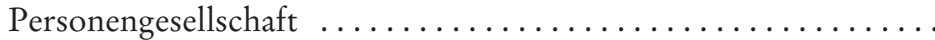

BARBARA GRUNEWALD

Stimmverbote nach $\$ 47$ Abs. 4 GmbHG

ohne Interessenkonflikt? . . . . . . . . . . . . . . . . . . . .

Mathias Habersack

Der Unterordnungskonzern - Terra Cognita? .............

Stephan Harbarth

Weisungsrecht und Berichtswege bei der Konzernleitung in Matrixstrukturen ..............................

Heribert Heckschen

Heilung unwirksamer Abfindungsklauseln ...............

Andreas Heidinger

Und sie dreht sich doch!

Ein Plädoyer für den gutgläubigen Erwerb des aufschiebend

bedingt abgetretenen Geschäftsanteils ...................

Joachim Hennrichs

Nichtigkeit des Jahresabschlusses und Auswirkungen auf Folgeabschlüsse und Gewinnverwendung $\ldots \ldots \ldots \ldots \ldots$

Martin Henssler

Gemischte Unternehmensgegenstände von Personengesellschaften ...

Godehard Kayser

Die Anfechtung als unentgeltliche Leistung - eine Allzweckwaffe des Insolvenzverwalters zur Massegenerierung? ............

Roger Kiem

Der Zweite Senat als Ersatzgesetzgeber .................. 


\section{Detlef KLEINDiEK}

Einziehung und Abfindungszahlung in der Rechtsprechung

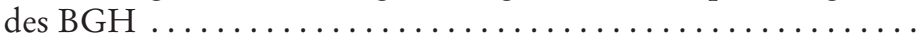

Michael Kling

Managerhaftung für Kartellverstöße bei Maßnahmen

des Informationsaustauschs mit Wettbewerbern ............

JENS KoCH

Die Legal Judgment Rule: ein Fall für die Wolfsschlucht ....... 413

JOACHIM KumMER

Gibt es unanfechtbare, aber gleichwohl nicht durchsetzbare

Darlehenssicherheiten? ...........................

Katja Langenbucher

Die Einflussnahme von Aktionären auf die Zusammensetzung des Vorstands.

Dieter Leuering

Einstweiliger Rechtsschutz gegen die Ausführung noch zu fassender Gesellschafterbeschlüsse . . . . . . . . . . . . . .

JAN LIEDER

Formbedürftigkeit der Kapitalerhöhung und verbundener Geschäfte $\ldots \ldots \ldots \ldots \ldots \ldots \ldots \ldots \ldots$

Hanno Merkt

Anmerkungen zur Treuepflicht des Kleinaktionärs

Peter O. Mülbert

EU-rechtliche Kapitalmarktinformationsvorschriften und mitgliedstaatliche Haftungsregeln - Möglichkeiten und Grenzen am Beispiel der Prospektverordnung (EU) 2017/1129 . . . . . . . .

Andreas Pentz

Differenzhaftung und verdeckte Mischeinlage/ verdeckte gemischte Sacheinlage $\ldots \ldots \ldots \ldots \ldots \ldots \ldots \ldots$

Markus Roth

Kommunikation des Aufsichtsrats

Volker SAnder

Die Entwicklung der Rechtsprechung zum Zahlungsverbot . . . . . 
INGO SAENGER

Treugebundene Zustimmungspflichten im GmbH-Recht . . . . . . 603

Carsten Schäfer

Neue Impulse für den Minderheitenschutz gegen

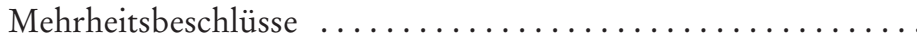

JessicA SCHMidT

Die Entwicklung des Handels- und Unternehmensregisterrechts in der EU im Spannungsfeld von Publizität, Geheimhaltungsinteressen und Persönlichkeitsrecht .........

KARSTEN SCHMIDT

$\mathbb{} 166 \mathrm{HGB}$ und das geltende Recht $\ldots \ldots \ldots \ldots \ldots \ldots \ldots$

Uwe H. Schneider und Sven H. Schneider

Organhaftung bei Tochtergesellschaften in der Rechtsform

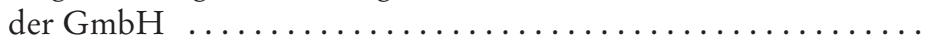

Ulrich SEIBERT

The long and winding road to the online registration of companies

Christoph H. SeibT

Vertrauensentzug durch Hauptversammlung oder Aufsichtsrat als wichtige Gründe der Vorstandsabberufung $\ldots \ldots \ldots \ldots \ldots$

Gerald Spindler

Personalunion in einem Konzern mit monistisch strukturierter SE

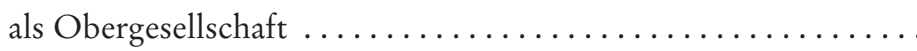

Lutz STrohn

Geht nicht - geht doch: Warum es für die Lösung des Problems „Einziehung und Abfindung“ 59 Jahre brauchte ...........

Christoph Teichmann

Die Verantwortung des GmbH-Geschäftsführers

für Gesellschafterliste und Transparenzregister $\ldots \ldots \ldots \ldots \ldots$

RÜDIGER VEIL

Maximalharmonisierung und mitgliedstaatliche Gesetzgebung im europäischen Marktmissbrauchsrecht $\ldots \ldots \ldots \ldots \ldots \ldots$ 
Dirk A. Verse

Zur Enthaftung der Geschäftsführer bei Befolgung von Gesellschafterbeschlüssen

Überlegungen zu $\int 43$ Abs. 3 Satz 3 GmbHG

EBERHARd VETTER

Unternehmensexterne als Versammlungsleiter

der Hauptversammlung

JOCHEN VETTER

Intuition und Business Judgment $\ldots \ldots \ldots \ldots \ldots \ldots \ldots \ldots$

Frauke WedEMANN

10 Jahre MoMiG: Die GmbH-Reform im Spiegel

der Rechtsprechung des II. Zivilsenats des BGH ..........

SimON WEILER

Der Schutz vor Fremdeinfluss auf Sportkapitalgesellschaften -

Satzungsgestaltung im deutschen Profifußball

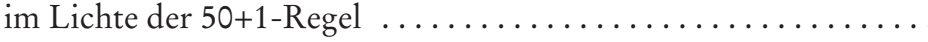

JohanNES WERTENBRUCH

Pfändung und Insolvenzbeschlag vinkulierter Namensaktien ....

Heinz WÖstManN

Kita-Rechtsprechung des BGH und Gewinnausschüttung ......

Hildegard Ziemons

Geschäftsleiterhaftung in der Eigenverwaltung $\ldots \ldots \ldots \ldots . \ldots 23$

Veröffentlichungen von VRiBGH a.D. Prof. Dr. Alfred Bergmann . . 937

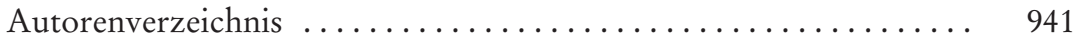


